Association for Information Systems AIS Electronic Library (AISeL)

Wirtschaftsinformatik Proceedings 2001

Wirtschaftsinformatik

September 2001

\title{
Erfolgswirkungen von One-to-One Marketing - Eine empirische Analyse
}

Dietmar Eifert

Albert-Ludwigs-Universität Freiburg, eifert@iig.uni-freiburg.de

Ingo Pippow

Albert-Ludwigs-Universität Freiburg, pippow@iig.uni-freiburg.de

Follow this and additional works at: http://aisel.aisnet.org/wi2001

\section{Recommended Citation}

Eifert, Dietmar and Pippow, Ingo, "Erfolgswirkungen von One-to-One Marketing - Eine empirische Analyse" (2001). Wirtschaftsinformatik Proceedings 2001.21.

http://aisel.aisnet.org/wi2001/21

This material is brought to you by the Wirtschaftsinformatik at AIS Electronic Library (AISeL). It has been accepted for inclusion in Wirtschaftsinformatik Proceedings 2001 by an authorized administrator of AIS Electronic Library (AISeL). For more information, please contact elibrary@aisnet.org. 
In: Buhl, Hans Ulrich, u.a. (Hg.) 2001. Information Age Economy; 5. Internationale Tagung Wirtschaftsinformatik 2001. Heidelberg: Physica-Verlag

ISBN: 3-7908-1427-X

(C) Physica-Verlag Heidelberg 2001 


\title{
Erfolgswirkungen von One-to-One Marketing - Eine empirische Analyse
}

\author{
Dietmar Eifert, Ingo Pippow \\ Albert-Ludwigs-Universität Freiburg
}

\begin{abstract}
Zusammenfassung: One-to-One Marketing ist ein neues Konzept für eine Steigerung der Wettbewerbsfähigkeit von Unternehmen, das über bisherige Marketingkonzepte wie Mass Customization etc. hinausgeht. Konzept und Wirkungsweise des One-to-One Marketing im E-Commerce werden vorgestellt. Anhand einer repräsentativen Studie zu Unternehmensaktivitäten im E-Commerce im deutschsprachigen Raum wird überprüft, inwiefern Unternehmen tatsächlich ihre Wettbewerbsfähigkeit durch One-to-One Marketing steigern können. Es zeigt sich, dass die Potentiale des One-to-One Marketing gegenwärtig noch nicht voll ausgenutzt werden.
\end{abstract}

Schlüsselworte: Electronic Commerce; One-to-One Marketing; Relationship Marketing; Customized Marketing; Faktorenanalyse; Clusteranalyse

\section{One-to-One Marketing im E-Commerce}

Die dauerhafte Bindung von Kunden an Unternehmen wird im E-Commerce immer mehr zum Problem. Dies liegt vor allem an den sinkenden Informationskosten für Konsumenten [Bako97], der folglich steigenden Markttransparenz und leichteren Abwanderung des Kundenstamms. Andererseits sorgen sinkende Informationskosten für eine Überflutung der Konsumenten mit irrelevanten Informationen, so dass Informationsfilter benötigt werden. Die Strategie von Unternehmen zur Bindung ihrer Kunden im E-Commerce sollte daher beinhalten, Kunden durch einen solchen Filter einen Mehrwert anzubieten. Zeitersparnis oder erhöhte Bequemlichkeit für Kunden können beispielsweise gewinnbringend eingesetzt werden [SmBa99].

Als Maßnahme zur Kundenbindung im E-Commerce wird u.a. das Konzept des One-to-One Marketing diskutiert [PeRo97], die einzelkundenorientierte Ausrichtung aller Marktaktivitäten mit dem Ziel der jeweils individuellen Ansprache und individuellen Problemlösung für Kunden [StSc99]. Hierbei geht es darum, unter Ausnutzung moderner Informations- und Kommunikationstechnologie ohne Medienbrüche Kunden mit individuell zugeschnittenen Produkten unmittelbar und 
individuell anzusprechen. One-to-One Marketing erfolgt durch die gleichzeitige Kombination von zwei Ansätzen zur Kundenbindung [Hild97]:

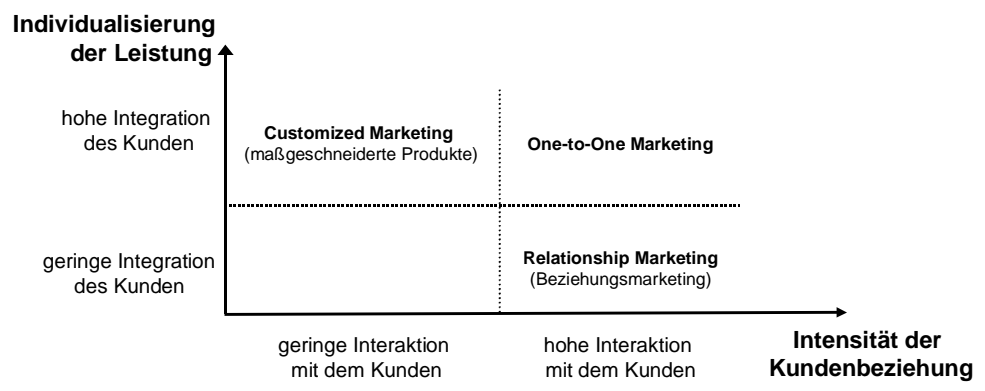

Abbildung 1: Kundenbindung durch One-to-One Marketing (in Anlehnung an [Hild97])

- Individualisierte Kundenansprache bzw. Relationship Marketing: Beim Relationship Marketing werden Kunden individuell und interaktiv angesprochen und es wird eine intensive Anbieter-Nachfrager Beziehung aufgebaut.

- Individualisierte Leistungserstellung bzw. Customized Marketing: Beim $\mathrm{Cu}$ stomized Marketing findet im Gegensatz zum Massenmarketing oder konventionellen Direktmarketing durch die hohe Integration des Kunden in den Leistungserstellungsprozess eine individuelle Leistungserstellung statt.

Im folgenden wird untersucht, inwiefern Unternehmen, die One-to-One Marketing einsetzen, bzgl. Kundenbindung erfolgreicher sind als andere Unternehmen. Dazu wird im folgenden Kapitel der Wirkungsprozeß der einzelnen Marketing Konzepte untersucht und anschließend anhand einer empirischen Studie getestet.

\section{Erfolgswirkung von One-to-One Marketing}

\subsection{Relationship Marketing}

Durch Relationship Marketing sollen Kunden langfristig an Unternehmen gebunden und Umsätze pro Kunde über deren gesamten Lebenszyklus hinweg maximiert werden. Als Mittel hierfür wird eine intensive, individuelle Kundenbeziehung aufgebaut - eine „learning relationship“ [DwSc87; PeRo97; PiPe95].

Beim Erstkauf gibt der Kunde Daten über seine Präferenzen preis, z.B. durch Angabe persönlicher Interessen. Diese Daten können zur Profilbildung genutzt werden, so dass der Kunde später individueller angesprochen werden kann. Bei Folgekäufen kann auf eine stetig verbesserte Datenbasis zurückgegriffen werden, was 
eine immer bessere Profilbildung erlaubt, so dass der Preis zur Generierung von Wiederkäufen sinkt. Aus dieser sich im Zeitablauf entwickelnden ,learning relationship“ können beide Seiten Gewinne erzielen [Pill00]: Anbieter lernen die Präferenzen und Zahlungsbereitschaften ihrer Kunden besser kennen und können dadurch solche Produkte verkaufen, die Kunden tatsächlich wünschen; Kunden reduzieren die Opportunitätskosten potenzieller Fehlkäufe aufgrund unklarer Präferenzbeschreibungen, da der Anbieter im Interesse einer langfristigen Beziehung keine unerwünschten Angebote machen wird.

Das Ziel der Kundenbindung durch Etablierung einer „learning relationship“ kann auf freiwilliger und auf unfreiwilliger Basis erreicht werden. Freiwillige Bindung eines Kunden an ein Unternehmen, bzw. Kundenloyalität, ist von mehreren Faktoren abhängig, wobei die Kundenzufriedenheit die entscheidende Bezugsgröße ist [BaHu98; JoSa95; HoGi99]. Die Kundenzufriedenheit wiederum steigt, wenn durch Erfüllung der Präferenzen des Kunden dessen Erwartungen an den Anbieter (über-) erfüllt werden [BaGr99]. Dies führt zu:

H1: Unternehmen, die Relationship Marketing betreiben, können die Zufriedenheit ihrer Kunden erhöhen.

Unfreiwillige Kundenbindung ist die Folge von Wechselkosten für Kunden, die diesen an der Abwanderung zu einem alternativen Anbieter hindern. Unternehmen können versuchen, sobald sie eine Kundenbeziehung aufgebaut haben, folgende Arten von Wechselkosten zu etablieren [ToDi00]:

- Direkte Wechselkosten, d.h. Opportunitätskosten in Form von Such- und Informationskosten bei einer Transaktion mit einem unbekannten Anbieter: Generell sinken diese z.B. durch Informationsdienste im Internet [Bako97]. Anbieter können jedoch versuchen, Opportunitätskosten der Informationssuche bei anderen Anbietern im E-Commerce zu erhöhen durch Maßnahmen wie Presale Services (ausführliche Informationen über Produkteigenschaften, Zahlungsbedingungen, Lieferbedingungen, etc.), durch die Kunden von der Suche alternativer Anbieter abgehalten werden können und After-sale Services wie Beschwerdemanagement oder technische Hilfe.

H2: Unternehmen, die Relationship Marketing betreiben, setzen Pre- und After-sale Services zur Erhöhung der direkten Wechselkosten ein.

- Lernkosten, d.h. der Verlust bisheriger Produkterfahrungen bei Wechsel des Anbieters: Diese hängen entscheidend von der Spezifität eines Produktes ab [HoGi99]. Anbieter von Standardprodukten werden kaum Lernkosten aufbauen können. Das Internet bietet jedoch die Möglichkeit, durch interaktive Abfragen oder spezielle Produktkonfiguratoren das „Erlernen“ der speziellen Umgebung eines Anbieters erforderlich zu machen.

H3: Unternehmen, die Relationship Marketing betreiben, setzen spezielle Produktkonfiguratoren zur Erhöhung der Lernkosten ein. 
- „Künstliche“ / vertragliche Wechselkosten, d.h. monetäre Anreize für Kunden, den Anbieter nicht zu wechseln: Hierzu zählen vor allem Rabatt- und Bonusprogramme, die neben der unmittelbaren Anreizwirkung beim Anbieter zu verbleiben den zusätzlichen Vorteil bieten, dass der Anbieter über die Teilnahme am Prämienprogramm Kundeninformationen erhält, die wiederum die Beziehung verbessern. Auch durch Gewinnspiele besteht ein monetärer Anreiz sich mit einem spezifischen Anbieter auseinanderzusetzen.

H4: Unternehmen, die Relationship Marketing betreiben, setzen Rabatt- und Bonusprogramme und Gewinnspiele zur Erhöhung künstlicher Wechselkosten ein.

- Sozialpsychologische Wechselkosten, d.h. Vertrauen und Commitment eines Kunden ggü. einem Anbieter [BaGr99]. Vertrauen entsteht, wenn sich Anbieter und Nachfrager nicht opportunistisch, sondern im gemeinsamen, langfristigen Interesse verhalten. Commitment oder eine ,innere Verbindung“ [DiKu98] des Kunden zum Anbieter bedeutet, dass der Kunde einen Anreiz hat, z.B. durch Informationsaustausch in die Beziehung mit dem Anbieter zu investieren. Sozialpsychologische Wechselkosten können im Internet erhöht werden durch die Kreierung von Communities. Dies gilt insbesonders für spezifische Themen oder geschlossene Kreise, bei denen eine Kontaktaufnahme zu Gleichgesinnten schwierig erscheint.

H5: Unternehmen, die Relationship Marketing betreiben, setzen ihre Vertrauenswürdigkeit oder Communities zur Erhöhung sozialpsychologischer Wechselkosten ein.

\subsection{Customized Marketing}

Ziel des Customized Marketing ist eine individuelle Leistungsfertigung nach den Wünschen jedes einzelnen Kunden [Schn97]. Der Kunde wird in den Produktionsprozeß integriert, um seine Präferenzen bereits dort berücksichtigen zu können.

Zur Integration des Kunden muß zunächst ein Kommunikationskanal zwischen Kunde und Unternehmen etabliert werden. Dieser Kommunikationskanal ist zweckgebunden für Informationsaustausch zum Ziel der individuellen Leistungserstellung. Der Kunde offenbart seine Präferenzen bzgl. des gewünschten Produktes, so dass dem Unternehmen die wesentlichen Produktcharakteristika bekannt sind. Anschließend wird das individuelle Produkt für den Kunden angefertigt [GiPi97].

Entscheidend für das Customized Marketing ist, dass der Kunde aktiv in den Leistungserstellungsprozess eingreift und daraus ein für ihn befriedigendes Ergebnis resultiert. Durch die Maßfertigung wird ein unmittelbarer Wettbewerbsvorteil realisiert, denn der Kunde erhält ein seinen Präferenzen entsprechendes Produkt.

Customized Marketing kann nur dann erfolgreich sein, wenn der Kunde einen Anreiz besitzt den Kommunikationskanal zum Unternehmen auch zu nutzen. Unter- 
nehmen müssen daher ihr Web-Angebot dahingehend ausweiten, dass sie im Rahmen des Customized Marketing Ansatzes den Nutzen individueller Produktgestaltung klar kommunizieren.

H6: Unternehmen, die Customized Marketing betreiben, setzen eine intensive Informationspolitik ein, um den unmittelbaren Nutzen individualisierter Produkte herauszustellen.

\subsection{One-to-One Marketing}

Ziel des One-to-One Marketing ist eine Umsatzsteigerung bei jedem einzelnen Kunden, dem möglichst viele Produkte über einen langen Zeitraum hinweg verkauft werden sollen [PeRo97]. Marketing Aktivitäten konzentrieren sich nicht mehr auf die Produkte, sondern auf Kunden, zu denen eine individuelle Beziehung mit individuellen Produktangeboten aufgebaut wird. Auch Marktanteilssteigerungen treten in den Hintergrund.

Die entscheidenden Schritte im Ablauf des One-to-One Marketing sind die Kontaktaufnahme mit Kunden, die fortlaufende individuelle Ansprache, Identifizierung und Segmentierung der Kunden und die fortlaufende Profilbildung.

Durch One-to-One Marketing erwirbt sich ein Unternehmen einen profitablen Kundenstamm und damit einen Wettbewerbsvorteil [PiPe95]. Unternehmen, die One-to-One Marketing betreiben, müßten daher insgesamt erfolgreicher bzw. profitabler im E-Commerce agieren als andere Unternehmen. Derartige „harte“ Profitabilitätskennzahlen sind jedoch sowohl schwer meß- bzw. vergleichbar als auch - aufgrund der dynamischen Kosten-Nutzen Relation - zeitabhängig. Daher wird zur Erfolgsmessung auf „weiche“ Faktoren zurückgegriffen, also auf die Erreichung von Teilerfolgen wie z.B. erhöhter Kundenzufriedenheit etc.

H7: Unternehmen, die One-to-One Marketing betreiben, agieren erfolgreicher im E-Commerce als andere Unternehmen.

\section{Empirische Ergebnisse}

\subsection{Die empirische Studie ,e-Reality 2000“}

Im Juni 2000 haben das Institut für Informatik und Gesellschaft der Universität Freiburg, Consulting Partner und Heyde AG Führungskräfte (zu mehr als 70\% aus den obersten Führungsebenen) in Deutschland, Österreich und der Schweiz durch das Marktforschungsunternehmen Infratest Burke zum Thema E-Commerce befragt. Gegenstand der Studie ,e-Reality 2000“ war der betriebswirtschaftliche 
Nutzen von E-Commerce aus Unternehmenssicht, unter Berücksichtigung von Fragestellungen wie den Hürden, Auswirkungen sowie strategischen Implikationen des Einsatzes von E-Commerce. Insgesamt wurden in einer repräsentativen Erhebung (Infratest Burke Arbeitsstätten Master Sample) 1.308 Unternehmensvertreter in Einzelinterviews mit 204 Detailfragen konfrontiert [StSc00].

\subsection{Strukturierung des Datenmaterials}

In der hier gewählten Vorgehensweise wird zur Dimensionsreduktion der Daten eine Clusteranalyse durchgeführt, um die Unternehmen entsprechend Abb. 1 bzgl. der unterschiedlichen Ausprägung ihrer Marketing Aktivitäten zu gruppieren. Vorangestellt wird eine Faktorenanalyse, um die Unabhängigkeit der gewählten Faktoren zu sichern.

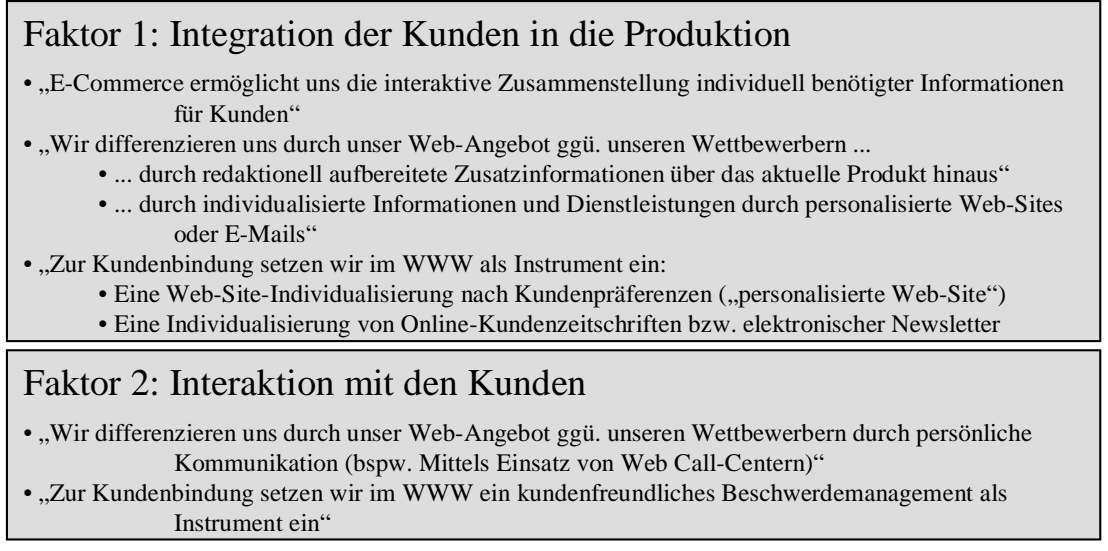

Abbildung 2: Faktoren, die die Marketing Aktivitäten bestimmen

Die Unternehmen wurden in insgesamt sieben Fragen direkt zu ihren Aktivitäten bzgl. One-to-One Marketing befragt. In der zugrunde liegenden Faktorenanalyse [BaEr96] werden zur Vermeidung von Verzerrungen nur diejenigen Fälle berücksichtigt, bei denen alle sieben Fragen beantwortet wurden $(n=543)$. Es können zwei Faktoren extrahiert werden (Kaiser-Kriterium), die sich wie in Abb. 2 (Faktorlösung nach Varimax-Rotation) interpretieren lassen. Mit einer erklärten Gesamtvarianz von 40,6\% ergeben die beiden Faktoren ein gutes Erklärungsmodell. Das Ergebnis der Faktorenanalyse, eines explorativen Verfahrens, bestätigt somit im Rahmen der zugrunde liegenden Fragen die theoretische Erwartung, dass die zwei Faktoren „Kundenintegration“ und „Kundeninteraktion“ die relevanten Dimensionen des One-to-One Marketing sind.

Die Verteilung der Unternehmen auf die unterschiedlichen Marketing Ausprägungen wird anschließend in einer (hierarchischen) Clusteranalyse über die beiden 
Faktoren Kundenintegration und Kundeninteraktion untersucht (Ward-Methode) [BaEr96]. Es werden vier Cluster gebildet (Elbow-Kriterium), so daß die einzelnen Cluster unmittelbar der Matrix aus Abb. 1 zugeordnet werden können:

\begin{tabular}{|l|l|l|l|}
\hline Cluster: & $\begin{array}{l}\text { Ausprägung Faktor } \\
\text { Kundenintegration }\end{array}$ & $\begin{array}{l}\text { Ausprägung Faktor } \\
\text { Kundeninteraktion }\end{array}$ & $\begin{array}{l}\text { Anzahl } \\
\text { Fälle }\end{array}$ \\
\hline $\begin{array}{l}1 \text { (Unternehmen mit One-to- } \\
\text { One Marketing) }\end{array}$ & Hoch & Hoch & 69 \\
\hline $\begin{array}{l}2 \text { (Unternehmen mit Relation- } \\
\text { ship Marketing) }\end{array}$ & Hoch & Niedrig & 195 \\
\hline $\begin{array}{l}3 \text { (Unternehmen ohne individu- } \\
\text { alisiertes Marketing) }\end{array}$ & Niedrig & Niedrig & 110 \\
\hline $\begin{array}{l}4 \text { (Unternehmen mit Custo- } \\
\text { mized Marketing) }\end{array}$ & Niedrig & Hoch & 169 \\
\hline
\end{tabular}

Tabelle 1: Marketing Aktivitäten der untersuchten Unternehmen

Im folgenden werden die bisher gebildeten Hypothesen dahingehend überprüft, ob sich die Unternehmen, die den jeweiligen Clustern zugeordnet wurden, tatsächlich annahmegemäß signifikant von den jeweils anderen Clustern unterscheiden. Hierzu werden jeweils einfaktorielle Varianzanalysen mit Scheffé-Test durchgeführt. Dieser Test ist relativ konservativ, also zu Gunsten der Nullhypothese [Bort99].

\section{3 Überprüfung der Hypothesen}

H1: Unternehmen, die Relationship Marketing betreiben, können die Zufriedenheit ihrer Kunden erhöhen.
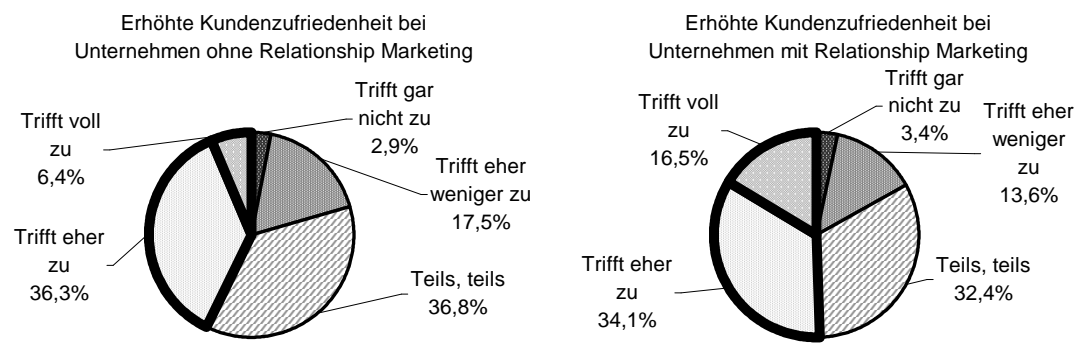

Abbildung 3: Gesteigerte Kundenzufriedenheit durch Relationship Marketing

Die Unternehmen wurden direkt nach der Veränderung der Zufriedenheit ihrer Kunden als Folge des E-Commerce Engagements des jeweiligen Unternehmens befragt. Eine erhöhte Kundenzufriedenheit konnten 42,7\% der Unternehmen fest- 
stellen, die kein Relationship Marketing betreiben (Summe aus „stimme zu“, „stimme voll zu“). Unternehmen, die Relationship Marketing betreiben, konnten jedoch zu 50,6\% eine erhöhte Zufriedenheit feststellen [Abb. 3]. Diese Werte unterscheiden sich signifikant $(\alpha=0,01)$, so dass $\mathrm{H} 1$ bestätigt wird.

\section{H2: Unternehmen, die Relationship Marketing betreiben, setzen Pre- und After-sale Services zur Erhöhung der direkten Wechselkosten ein.}

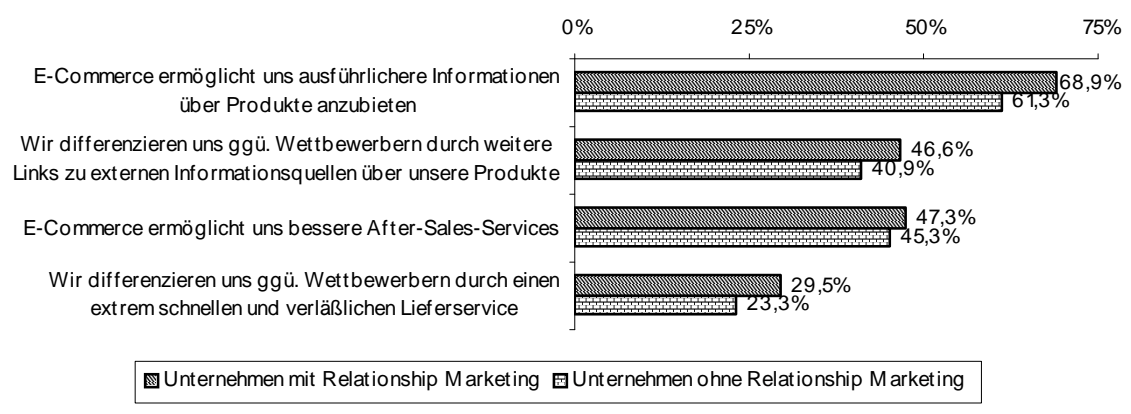

Abbildung 4: Pre- und After-sale Services der Unternehmen

Den Unternehmensvertretern wurden Fragen über die Möglichkeiten der Informationsbereitstellung für Kunden vor - und der Kundenbetreuung nach - einem $\mathrm{Ge}-$ schäftsabschluss gestellt. So gaben 61,3\% der Unternehmen, die kein Relationship Marketing einsetzen, an, E-Commerce ermögliche ausführlichere Informationen und Beratung über Produkte oder Dienstleistungen anzubieten [Abb. 4]. Signifikant höher $(\alpha=0,01)$ war hier die Angabe der Unternehmen, die Relationship Marketing anbieten, mit 68,9\%. Fragen zu After-sale Services wie z.B. eines schnellen, verlässlichen Lieferservice ergaben nur zufällige Unterschiede, so dass nur der erste Teil der Hypothese angenommen werden kann.

\section{H3: Unternehmen, die Relationship Marketing betreiben, setzen spezielle Produktkonfiguratoren zur Erhöhung der Lernkosten ein.}

Die Unternehmensvertreter wurden nach den wesentlichen Differenzierungsmerkmalen ihres Web-Angebotes gegenüber ihren Wettbewerbern gefragt. Unternehmen ohne Relationship Marketing gaben nur zu 15,8\% an, dass Service-Programme wie Kostenkalkulatoren oder Produktkonfiguratoren Bestandteil ihres WebAngebotes sind. Signifikant höher $(\alpha=0,01)$ war mit $24,6 \%$ die Antwort bei Unternehmen mit Relationship Marketing [Abb. 5]. Aufgrund des absolut niedrigen Wertes kann die Hypothese nur bedingt angenommen werden. 


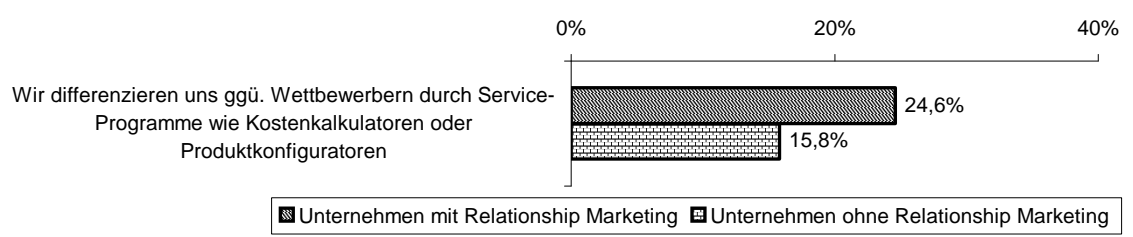

Abbildung 5: Erhöhung der Lernkosten durch Service-Programme

H4: Unternehmen, die Relationship Marketing betreiben, setzen Rabatt- und Bonusprogramme und Gewinnspiele zur Erhöhung künstlicher Wechselkosten ein.

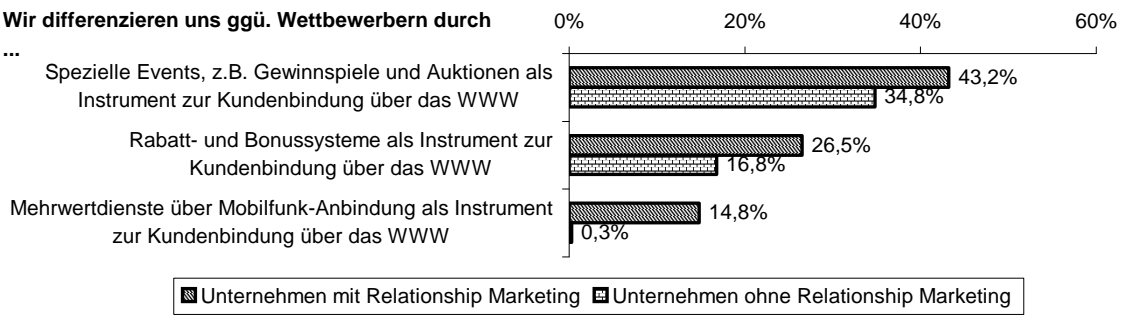

Abbildung 6: Kundenbindungsinstrumente im WWW

Die Unternehmen wurden nach verschiedenen preispolitischen Instrumenten zur Kundenbindung über das WWW gefragt. Den Einsatz von Rabatt- und Bonussystemen gaben $16,8 \%$ der Unternehmen ohne Relationship Marketing an. Signifikant höher $(\alpha=0,01)$ war der Einsatz bei Unternehmen mit Relationship Marketing in 26,5\% der Fälle [Abb. 6]. Ähnliches ergab sich auch bei der Frage, ob spezielle Events, wie z.B. Gewinnspiele oder Auktionen zur Kundenbindung eingesetzt werden. Der Unterschied zwischen Unternehmen ohne Relationship Marketing $(34,8 \%)$ und mit $(43,2 \%)$ war signifikant $(\alpha=0,01)$. Nicht bestätigt wird, dass diese Instrumente auch zur Datengewinnung und Profilerstellung genutzt werden. $\mathrm{H} 4$ wird aufgrund der absolut niedrigen Werte bedingt angenommen.

H5: Unternehmen, die Relationship Marketing betreiben, setzen ihre Vertrauenswürdigkeit oder Communities zur Erhöhung sozialpsychologischer Wechselkosten ein.

$\mathrm{Zu}$ den weiteren Differenzierungsmerkmalen, nach denen Unternehmensvertreter befragt wurden, zählen Communities bzw. Funktionalitäten wie z.B. Chats, die Gleichgesinnte zusammenführen. Unternehmen ohne Relationship Marketing bo- 
ten dies zu 0,1\% (mit: zu 18,9\%) an [Abb. 7]. Vertrauenswürdigkeit signalisierten die Unternehmen ohne Relationship Marketing durch ein hohes technisches Sicherheitsniveau zu 15,8\% (mit: 31,4\%), durch vertrauensbildende Signale und Maßnahmen wie z.B. Gütesigel und „Trusted Shops“ zu 11,9\% (23,5\%) oder durch kundenfreundlichen Datenschutz bzw. das Versprechen, Kundeninformationen nicht an Dritte weiterzugeben, zu 34,0\% (57,8\%). Alle Unterschiede waren signifikant $(\alpha=0,01)$. Die Hypothese wird bedingt angenommen.

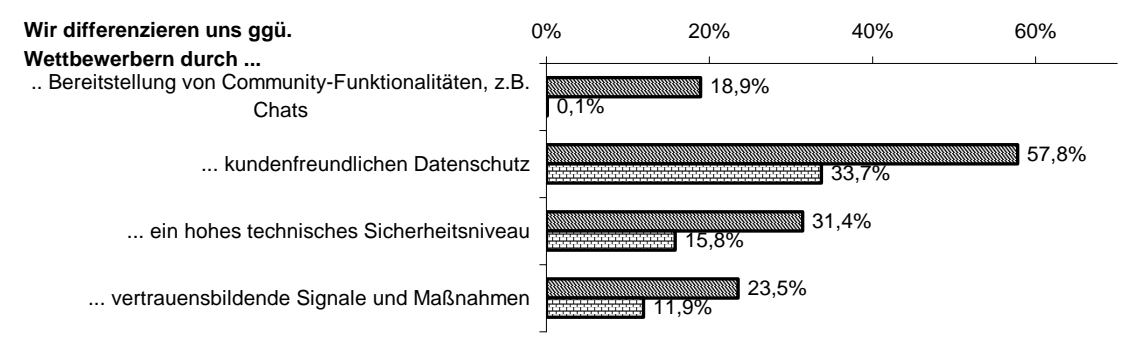

田Unternehmen mit Relationship Marketing BUnternehmen ohne Relationship Marketing

Abbildung 7: Vertrauensbildende Maßnahmen im E-Commerce

H6: Unternehmen, die Customized Marketing betreiben, setzen eine intensive Informationspolitik ein, um den unmittelbaren Nutzen individualisierter Produkte herauszustellen.

Die Informationspolitik der Unternehmen wurde durch zwei Fragen erfasst. Zum einen wurden die Unternehmen befragt, ob Links zu externen Informationsquellen über ihre Produkte (z.B. Warentests) Bestandteil des Web-Angebotes seien. Dies gaben 36,7\% der Unternehmen an, die kein Customized Marketing betreiben und signifikant $(\alpha=0,01)$ höher $52,5 \%$ der Unternehmen, die Customized Marketing betreiben. Zum anderen wurde gefragt, ob E-Commerce den Unternehmen ermöglicht, ausführliche Informationen und Beratungen über Produkte oder Dienstleistungen anzubieten. Da sich hier keine signifikanten Unterschiede ergaben, kann H6 weder angenommen noch abgelehnt werden.

\section{H7: Unternehmen, die One-to-One Marketing betreiben, agieren} erfolgreicher im E-Commerce als andere Unternehmen.

Die Unternehmensvertreter wurden in der Studie direkt nach der Erreichung verschiedener Unternehmensziele wie z.B. erhöhter Kundenzufriedenheit oder Kundenloyalität gefragt. Die aus Abb. 8 erkennbare Tendenz einer gestiegenen Kundenzufriedenheit bzw. -loyalität ist allerdings nicht signifikant. Während zwar durch Relationship Marketing die Kundenzufriedenheit zunimmt [Abb. 3], kann 
diese durch die zusätzliche Kombination mit Customized Marketing nicht weiter gesteigert werden. Somit läßt sich zumindest derzeit noch nicht bestätigen, dass die Unternehmen das Ziel einer erhöhten Kundenzufriedenheit durch One-to-One Marketing tatsächlich erreichen. Dennoch zeigen sich erste Erfolge: So wurden die Unternehmen danach gefragt, ob ihnen das Internet eine effektive Kommunikation von Markenimage und Unternehmensidentität erlaube. Dabei ergab sich mit $83,6 \%$ für die Unternehmen mit One-to-One Marketing eine signifikant $(\alpha=0,01)$ höhere Zustimmung als bei den übrigen drei Unternehmertypen. Ferner zeigt sich, dass Unternehmen ohne eine ausgeprägte Individualisierung mit nur 35,9\% bisher signifikant $(\alpha=0,1)$ weniger neue Dienstleistungen im E-Commerce anbieten konnten als die übrigen Gruppen. Somit zeigt sich, dass die Unternehmen hinsichtlich ihrer Kommunikation mit Kunden oder ihres Innovationsgrades im ECommerce mit unterschiedlichem Erfolg voranschreiten. Es ist zu erwarten, dass sich dies zukünftig auch in unterschiedlichen Beurteilungen der Unternehmensvertreter hinsichtlich der Ziele Kundenzufriedenheit bzw. -loyalität niederschlagen wird.

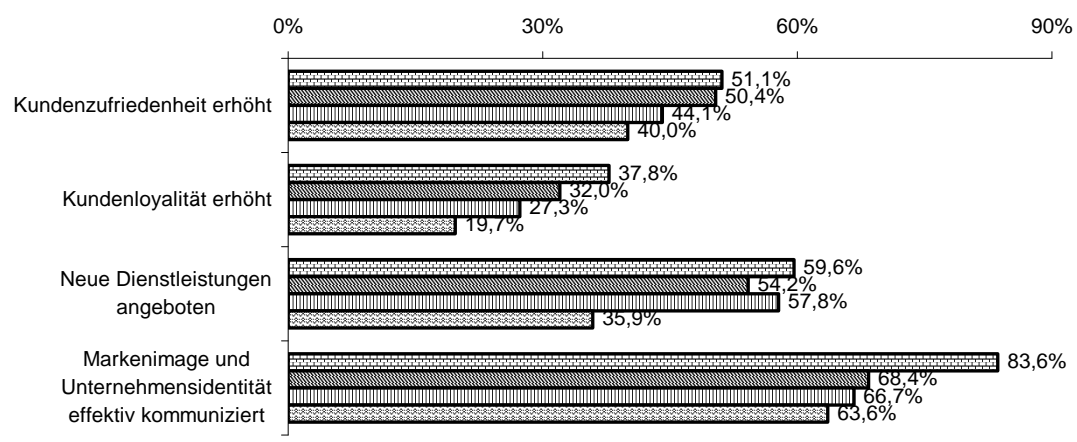

\begin{tabular}{|ll|}
\hline ⿴囗十 Unternehmen mit One-to-One Marketing & Unternehmen mit Relationship Marketing \\
囬Unternehmen mit Customized Marketing & QUnternehmen ohne individualisiertes Marketing
\end{tabular}

Abbildung 8: Erfolge im E-Commerce

\begin{tabular}{|l|c|c|}
\hline Cluster. & $\begin{array}{l}\text { "Internet ist für uns kein Me- } \\
\text { dium, um daraus nachhaltigen } \\
\text { ökon. Nutzen zu ziehen“ }\end{array}$ & $\begin{array}{l}\text { "Seitdem wir im Netz } \\
\text { sind, sind wir insge- } \\
\text { samt erfolgreicher“ }\end{array}$ \\
\hline 1 (One-to-One Marketing) & $\mathbf{8 2 , 1 \% \% ^ { 2 }}$ & $\mathbf{4 5 , 9 \%}$ \\
\hline 2 (Relationship Marketing) & $66,0 \%$ & $21,7 \%$ \\
\hline 3 (Kein individ. Marketing) & $72,0 \%$ & $21,6 \%$ \\
\hline 4 (Customized Marketing) & $67,1 \%$ & $24,3 \%$ \\
\hline
\end{tabular}

Tabelle 2: Jeweilige ${ }^{1}$ Zustimmung bzw. ${ }^{2}$ Ablehnung zu Erfolgsaussichten 
Auch die generellen Erfolgsaussichten der Unternehmen im E-Commerce wurden durch zwei Fragen abgedeckt. Ihre Verteilung auf die unterschiedlichen Gruppen ist in Tabelle $2 \mathrm{zu}$ sehen. Unternehmen, die One-to-One Marketing betreiben, sehen sich jeweils signifikant $(\alpha=0,01)$ besser ausgerichtet als die übrigen Unternehmen. Insgesamt läßt sich $\mathrm{H} 7$ damit bedingt annehmen.

\section{Gegenwärtige Umsetzungsprobleme des One-to- One Marketing}

One-to-One Marketing ist für die Anbieter mit hohen Risiken verbunden. Im Gegensatz zum Massenmarketing ist bereits die erste Kontaktaufnahme mit hohen Kosten verbunden, Erträge ergeben sich erst im Zeitablauf einer UnternehmenKunden-Beziehung. Dementsprechend müssen auch die Kosten für den Aufbau einer Kundenbeziehung über den entsprechenden Zeitraum betrachtet werden [DwSc87]. Das Risiko hoher, versunkener Kosten bei Abwanderung des Kunden nach einer geringen Anzahl von Transaktionen trägt somit allein der Anbieter. Auch die individualisierte Produkterstellung für einzelne Nachfrager kann mit hohen Kosten verbunden sein [Schn97]. Der den Kunden entstehende Zusatznutzen aufgrund der besseren Präferenzerfüllung muss daher in vernünftiger Relation zu den Produktionskosten stehen. So sollte ein Unternehmen die individualisierten Produkte und Leistungen in Massenproduktion erstellen können.

Für eine optimale Durchführung des One-to-One Marketing ist ferner ein kooperatives Verhalten des Kunden Voraussetzung. So könnte die Profilerstellung durch ein unkooperatives Verhalten der Kunden erschwert bzw. verfälscht werden. Der Kunde muss bereits beim Erstkauf bereit sein, Daten preis zu geben und einer fortlaufenden Datenerhebung gegenüber aufgeschlossen sein. Viele Kunden lehnen allerdings eine persönliche Beziehung zum Anbieter ab und wehren sich gegen eine Nutzung ihrer persönlicher Kundendaten, da sie daraus keinen unmittelbaren Nutzen erkennen [Pill00] und eine Vertrauensbasis zum Anbieter nicht vorhanden ist [StSc00]. Diese Vertrauensbasis zwischen Unternehmen und Kunden ist Vorraussetzung für ein funktionierendes One-to-One Marketing.

\section{Zusammenfassung und Ausblick}

Die Internet-Ökonomie zwingt die Unternehmen dazu, ihre Marketingstrategien zu überdenken. Mit Hilfe des One-to-One Marketing haben sie die Möglichkeit der größeren Kundenfluktuation im E-Commerce entgegenzuwirken. Voraussetzung dafür ist die Umsetzung von Relationship Marketing zur Personalisierung der Kommunikation und Customized Marketing zur individuellen Leistungserstellung. 
Mit Hilfe einer empirischen Studie wurden Hypothesen zu den Erfolgswirkungen der einzelnen Marketing Konzepte überprüft. Es zeigt sich, dass Relationship Marketing signifikant zur Erhöhung von Kundenzufriedenheit und zum Aufbau von Wechselkosten für Kunden im E-Commerce beiträgt. Die erwartete weitere $\mathrm{Zu}$ nahme von Kundenzufriedenheit durch eine individuelle Leistungserstellung konnte nicht bestätigt werden. Die für Customized Marketing nötige Umstrukturierung des Produktionsablaufes und dabei eventuell auftretende Probleme wurden jedoch in dieser Arbeit nicht berücksichtigt. Die eine effiziente Integration des Kunden in den Produktionsprozeß ist daher weiter zu erforschen.

Eine mögliche Interpretation der Ergebnisse liegt darin, dass Unternehmen, die bereits One-to-One Marketing einsetzen, zwar besser mit ihren Kunden kommunizieren können und durch innovative Leistungen bereits Maßnahmen zu einer erhöhten Kundenzufriedenheit umsetzen. Der nachhaltige Erfolg durch eine erhöhte Kundenbindung im E-Commerce hat sich aber aufgrund des insgesamt kurzen Zeithorizonts noch nicht einstellen können.

Es empfiehlt sich daher für Unternehmen, die Ausrichtung des Marketing und der Organisationsstruktur auf One-to-One Marketing hin zu prüfen, um steigendem Wettbewerbsdruck entgegenwirken zu können. Produktionstechnisch besteht hierfür bereits das Konzept der Mass Customization, bei der eine hohe Variantenvielfalt mit geringer Produktionsvielfalt erreicht wird. Gleichzeitig muß jedoch die Kommunikation mit Kunden intensiviert werden. Der Einsatz von Informationstechnologie muß ferner Datensicherheit ebenso gewährleisten wie eine vertrauliche Behandlung der Kundenprofile.

\section{Literatur}

[BaEr96] Backhaus, K.; Erichson, B.; Plinke, W.; Weiber, R. (1996): "Multivariate Analysemethoden. Eine anwendungsorientierte Einführung", 8. Aufl., Springer, Berlin.

[Bako97] Bakos, Y., (1997): "Reducing Buyer Search Costs: Implications for Electronic Marketplaces", Management Science, Vol. 43, No. 12, Dezember 1997, S. 1676-1692.

[BaGr99] Bauer, H.; Grether, M.; Leach, M. (1999): "Relationship Marketing im Internet", Jahrbuch der Absatz- und Verbrauchsforschung, 3, 1999, S. 284-302.

[BaHu98] Bauer, H.H.; Huber, F.; Betz, J. (1998): "Erfolgsgrößen im Automobilhandel. Ergebnisse einer kausalanalytischen Studie", Zeitschrift für Betriebswirtschaft, 68, 9, S. 979-999.

[Bort99] Bortz, J., (1999): "Statistik für Sozialwissenschaftler", 5. Auflage, Springer, Berlin.

[DiKu98] Diller, H.; Kusterer, M. (1988): "Beziehungsmanagement: Theoretische Grundlagen und explorative Befunde", Marketing ZFP, 3, S. 211-220. 
[DwSc87] Dwyer, R.F.; Schurr, P.H.; Oh Sejo (1987): "Developing Buyer-Seller Relationships", Journal of Marketing, 51, S. 11-27.

[GiPi97] Gilmore, H.; Pine, J.B. (1997): "The Four Faces of Mass Customization", Harvard Business Review, January-February, S. 91-101.

[Hild97] Hildebrand, V.D. (1997): "Individualisierung als strategische Option der Marktbearbeitung: Determinanten und Erfolgswirkungen kundenindividueller Marketingkonzepte", Gabler, Wiesbaden.

[HoGi99] Homburg, C.; Giering, A.; Hentschel, F. (1999): "Der Zusammenhang zwischen Kundenzufriedenheit und Kundenbindung", Die Betriebswirtschaft, 59, 2, S. 174-195.

[JoSa95] Johnes, T.O.; Sasser, W.E. (1995): "Why Satisfied Customers Defect", Harvard Bussines Review, S. 88-99.

[PeRo97] Peppers, D.; Rogers, M. (1997): "Enterprise one to one: Tools for competition in the interactive age", Doubleday, New York.

[Pill00] Piller, F.T. (2000): "Mass Customization: Ein wettbewerbsstragegisches Konzept im Informationszeitalter", Gabler, Wiesbaden.

[PiPe95] Pine, J.B.; Peppers, D.; Rogers, M. (1995): "Do You Want to Keep Your Customers Forever?", Harvard Business Review, March-April, S. 103-114.

[Schn97] Schnäble, P. (1997): "Mass Customized Marketing: Effiziente Individualisierung von Vermarktungsobjekten und -prozessen", Gabler, Wiesbaden.

[SmBa99] Smith, M.; Bailey, J.; Brynjolfsson, E. (1999): "Understanding Digital Markets: Review and Assesments".

[StSc99] Strauß, R.; Schoder, D. (1999): "Wie werden die Produkte den Kunden angepaßt? - Massenhafte Individualisierung", in: Sönke, A. et al. (Hrsg.): „eCommerce-Einstieg, Strategie und Umsetzung im Unternehmen", S. 109-119.

[StSc00] Strauß, R.; Schoder, D. (2000): "e-Reality 2000 - Electronic Commerce: Von der Vision zur Realität", 1, Consulting Partner Group, Frankfurt.

[ToDi00] Tomczak, T.; Dittrich, S. (2000): "Kundenbindung - Bestehende Kundenpotentiale nutzen", in: Hinterhuber, H. H. (Hrsg.): Kundenorientierte Unternehmensführung. 